\title{
Soliton trains in dispersive media
}

\author{
Jüri Engelbrecht, Tanel Peets, and Kert Tamm \\ Laboratory of Solid Mechanics, Department of Cybernetics, School of Science, Tallinn University of Technology \\ Akadeemia tee 21, Tallinn 12618, Estonia \\ E-mail: je@ioc.ee, tanelp@ioc.ee, kert@ioc.ee
}

Received December 19, 2017, published online May 28, 2018

\begin{abstract}
In this paper two Boussinesq-type mathematical models are described which lead to solitonic solutions. One case corresponds to microstructured solids, another case to biomembranes. The emergence of soliton trains in both cases is demonstrated by using numerical simulation. The pseudospectral method guarantees the high accuracy in computing. The significance of the nonlinearities - either deformation-type or displacement-type, is demonstrated.
\end{abstract}

PACS: 46.40.Cd Mechanical wave propagation (including diffraction, scattering, and dispersion);

47.35.Fg Solitary waves;

47.54.Fj Chemical and biological applications.

Keywords: dispersion, nonlinearities, microstructure, biomembranes, solitons.

To the memory of A.M. Kosevich

\section{Introduction}

The celebrated wave equation is one of the classical equations of mathematical physics and describes the motion of a wave with a constant speed. For many practical applications this model must be generalised. One of such a generalisation in conservative media is called after Boussinesq, who derived this model for surface waves on a fluid layer [1,2]. Nowadays the Boussinesq-type models are widely used also in solid mechanics [3]. In brief, such models are (i) bi-directional (including the d'Alembert operator); and in addition (ii) include nonlinear terms (of any order); (iii) include higher order terms (the presence of space and time derivatives of the fourth order or higher) describing the dispersive effects [3]. In general terms, the Boussinesq equation may be presented in a following form:

$$
u_{t t}-c_{0}^{2} u_{x X}+N(u)=D(u),
$$

where $u$ is the displacement, $c_{0}$ is the velocity and indices $x, t$ here and further denote differentiation. Operator $N(u)$ expresses nonlinear effects

$$
N(u)=N\left(u^{2}, u^{3}, \ldots, u u_{x}, \ldots\right)
$$

and $D(u)$ describes dispersive effects

$$
D(u)=D\left(u_{4 x}, u_{4 t}, u_{2 x 2 t}, u_{6 x}, \ldots\right) .
$$

There are many studies of this type of equations derived using various physical assumptions [3-7]. Attention is paid to the mathematical correctness of models in the sense of Hadamard, i.e., establishing whether the initial value problem is well-posed or ill-posed [3,5]. In physical terms, the Boussinesq-type models describe waves in crystals [5,8], longitudinal waves in rods [6,9], waves in microstructured solids [10,7], waves in biomembranes [11], etc.

The most remarkable phenomenon resulting from using models where nonlinearity and dispersion are both taken into account, is the possibility of existence and/or emerging of solitons. Many studies are devoted to the special type of dispersion when $D(u)=u_{x x x x}$. This is typical for cases when the governing equation is derived on the basis of lattice dynamics [5]. The "well-posedness" of such a model is analysed in detail $[12,13]$.

The Boussinesq-type equations which actually model weak dispersion, are not the only ones able to describe solitons, the sine-Gordon equation, for example, is able to model solitons and bound-soliton complexes [14] emerging in ferromagnets. The combination of the sine-Gordon and the Boussinesq-type equations permits to analyse the dislocation (crowdion) motion in crystals [15]. This model is

$$
u_{t t}-u_{x x}+\sin u-\gamma u_{x}^{2} u_{x x}-\beta u_{x x x x}=0,
$$

where $\gamma$ and $\beta$ are the physical parameters. Equation (4) is nowadays called the Kosevich-Kovalev equation describing 
motion in a strongly dispersive medium. Other generalisations are possible demonstrating the richness of the model [16]. The striking duality of solitons and quasi-particles is noted [17].

Based on this brief overview, it is clear that Boussinesq-type equations govern complicated dynamics. However, one should clearly describe the physics behind the mathematical models. In what follows, the focus is on the emergence of soliton trains modelled by the Boussinesq-type equations. The attention will be paid to the model equations focusing on the influence of various nonlinearities on the emergence process together with the fourth-order dispersive terms. The physical background of models is related to the microstructured media: microstructured Mindlin-type solids and biomembranes which possess internal structure. The latter is qualified also as a microstructure. In Sec. 2 types of nonlinearities (deformation-dependent and displacement-dependent) are presented and analysed. Section 3 is devoted to mathematical models with dispersive terms. In this case the inertia of a microstructure is taken into account which leads to dispersion operator $D(u)=D\left(u_{x x x x}, u_{x x t t}\right)$. This means that in mechanics of microstructured solids dispersion is more complicated than proposed in lattice dynamics [5]. The emergence of soliton trains for two model cases is analysed in Sec. 4. Finally, the discussion and conclusions are presented in Sec. 5. The results obtained earlier are here analysed from a unified viewpoint.

\section{Nonlinearities}

In mechanics of solids the nonlinearities are caused by physical and geometrical effects (see [18]). According to the conventional continuum theory [19], the physical nonlinearity means the nonlinearity of the stress-strain relations and the geometrical nonlinearity is related to the finite deformations, i.e., to the strain tensor. The free energy function (potential) in terms of the strain tensor is then presented in the form including beside quadratic terms also the higher order terms. Such a form reflects better the shape of the potential in terms of forces between the atoms in the discrete lattice. Note that as far as the stress tensors are determined by the derivatives of the potential with respect to the components of the deformation tensor, the quadratic terms lead to the conventional linear theory [19]. The first approximation of a nonlinear stress tensor includes the quadratic polynomial of displacement gradients. For example, in the one-dimensional case the Kirchhoff stress tensor is [18]

$K_{11}=(\lambda+2 \mu) u_{x}+\left(\frac{1}{2} \lambda+\mu+3 v_{1}+3 v_{2}+3 v_{3}\right) u_{x}^{2}+\ldots$,

where $\lambda, \mu$ are the elastic constants of the second order (the Lamé parameters) and $v_{1}, v_{2}, v_{3}$ are the elastic constants of the third order. It means that the velocity is determined by the relation

$$
c^{2}=c_{0}^{2}\left(1+k u_{x}\right)
$$

where $c_{0}$ is the velocity in the unperturbed state and $k$ is the constant of nonlinearity which according to expression (5) is $k=3\left(1+m_{0}\right), m_{0}=2\left(v_{1}+v_{2}+v_{3}\right)(\lambda+2 \mu)^{-1}$.

Such a situation may lead to the formation of shock waves, i.e., the discontinuities of the solution $[18,20]$. More complicated free energy potentials lead certainly to more complicated mathematical models [18] but the situation described briefly above is the fundamental case of the nonlinear wave motion in solids.

In biological tissues and cells, Exp. (6) might be different, especially when the discreteness of structures is taken into account. Such a situation is the case of biomembranes which are built by bilayers of lipid molecules. Based on experimental observations, Heimburg and Jackson [21] have proposed for longitudinal waves in biomembranes

$$
c^{2}=c_{0}^{2}+p u+q u^{2},
$$

where $u=\Delta \rho_{A}, \rho_{A}$ is the density and $p, q$ are experimentally determined constants.

It means that contrary to solids with deformationdependent $\left(u_{x}\right)$ nonlinearities, for biomembranes the governing wave equation based on expression (7) includes nonlinearities in terms not $u_{x}$ but simply $u$, i.e., the nonlinearities are of displacement-type (see below).

\section{Mathematical models for dispersive waves}

In general, dispersion of waves is the separation of waves into constituents of different wave-lengths and may be caused by either geometrical or physical effects. The geometrical dispersion takes place in waveguides due to the influence of the existence of lateral surfaces [9]. The physical dispersion in solids is caused by the existence of the microstructure of the material [5,7]. In the first case dispersion depends on the transverse dimensions of waveguides, in the second case - on the scale effects.

Here we present two nonlinear mathematical models where the physical dispersion is of importance. Both models are of the Boussinesq-type like Eq. (1).

Microstructured solids. In the theory of microstructured solids $[22,23]$ the behavior of the macro- and microcontinuum is described by the separate balance laws. In terms of macro-displacement $u$ and microdeformation $\varphi$, the simplest free energy $W$ is governed by a cubic function

$W=\alpha u_{x}^{2}+A \varphi u_{x}+\frac{1}{2} B \varphi^{2}+\frac{1}{2} C \varphi_{x}^{2}+\frac{1}{6} N u_{x}^{3}+\frac{1}{6} M \varphi_{x}^{3}$,

where $\alpha, A, B, C, N, M$ denote material parameters [10].

The balance laws are derived then from the EulerLagrange equations. Introducing dimensionless variables $X=x / L_{0}, T=c_{0} t / L_{0}, U=u / U_{0}$ where $c_{0}^{2}=\alpha / \rho$ and $U_{0}$ and $L_{0}$ are certain constants, along with geometrical pa- 
rameters $\delta=\left(l_{0} / L_{0}\right)^{2}$ and $\varepsilon=U_{0} / L_{0}$, where $l_{0}$ is the characteristic scale of the microstructure, the governing Boussinesq-type equation of motion is derived. By using the slaving principle (see [23]) this governing equation in terms of deformation $\left(V=U_{X}\right)$ is [24]

$$
\begin{gathered}
V_{T T}-b V_{X X}-\frac{\mu}{2}\left(V^{2}\right)_{X X}= \\
=\delta\left(\beta V_{T T}-v V_{X X} \frac{\lambda \sqrt{\delta}}{2}\left(V_{X}^{2}\right)_{X}\right)_{X X},
\end{gathered}
$$

where $b=1-A^{2} /(\alpha B), \mu=N U_{0} /\left(\alpha L_{0}\right), \beta=I A^{2} /\left(\rho l_{0}^{2} B^{2}\right)$, $v=C A^{2} /\left(\alpha B^{2} l_{0}^{2}\right)$ and $\lambda=A^{3} M U_{0} /\left(\alpha B^{3} l_{0}^{3} L_{0}\right)$ are constants. Here $I$ denotes microinertia.

This equation has a hierarchical structure [20] - two nonlinear wave operators (one at the l.h.s., another at the r.h.s.) describe motion in macro- and microstructure, respectively. In such a way, this is an explicit description of mechanical waves in microstructured media $[7,20]$ which takes into account the leading effects. The accuracy of this approximation is established by the analysis of dispersion relations of the original and approximated equations and depends on the ratio of velocities in macro- and microstructure [25]. Note also that even without the operator of the wave motion in the microstructure, the velocity of waves in the macrostucture is affected (see the structure of the coefficient $b$ ). It is possible to solve the inverse problem for Eq. (9) in order to determine the values of its coefficients with a suitable accuracy [26].

Equation (9) has soliton-type solutions if the following condition

$$
\left(\frac{\beta c^{2}-v}{c^{2}-b}\right)^{3}>\frac{4 \lambda^{2}}{\mu^{2}}
$$

is satisfied [26]. Here the velocity of a soliton $c$ is a free parameter. It is interesting to note that for solutions of the highly dispersive Kosevich-Kovalev equation (4) there are also restrictions which govern the existence of soliton complexes [16]. Such conditions seem to be characteristic to generalised models.

Biomembranes. These important building blocks for cells and nerves are built by lipid molecules which have hydrophobic tails directed inwards [25]. It has been demonstrated experimentally that such bilayers are able to carry mechanical waves [26,27]. The molecules of a bilayer can be treated as a microstructure and similarly to solids, the inertia of the microstructure must be taken into account.

The mathematical model for longitudinal waves in biomembranes including nonlinearity of the biomembrane expressed by Eq. (7) was derived by Heimburg and Jackson [21] and improved by Engelbrecht et al. [28] including also inertial effects. The governing equation in the dimensionless form is the following:

$$
\begin{gathered}
U_{T T}=\left(1+P U+Q U^{2}\right) U_{X X}+ \\
+(P+2 Q U) U_{X}^{2}-H_{1} U_{X X X X}+H_{2} U_{X X T T},
\end{gathered}
$$

where $X=x / l, T=c_{0} t / l, \quad U=u / \rho_{A}$ and $P=p \rho_{A} / c_{0}^{2}$, $Q=q \rho_{A}^{2} / c_{0}^{2}, \quad H_{1}=h_{1} /\left(c_{0}^{2} l^{2}\right), \quad H_{2}=h_{2} / l^{2}$. Note that $u=\Delta \rho_{A}$ and $l$ is a certain length (in case of a nerve fibre it is the fibre diameter). The constants $h_{1}$ and $h_{2}$ are dispersion parameters reflecting the elasticity and inertia of the structure, respectively.

The accounting of inertia (term $U_{X X T T}$ ) means that the propagation velocity is bounded for higher frequency harmonics [28]. Moreover, neglecting this term, i.e., the presence only the spatial fourth order derivatives in Eq. (10) can lead to instabilities of the solution [5].

\section{The emergence of soliton trains}

Both mathematical models presented in Sec. 3 are specific cases of the Boussinesq-type Eq. (1) including nonlinear and dispersive terms. As well it is known, under the certain conditions the nonlinear and dispersive effects could be balanced resulting in solitons. Since the pioneering studies of Zabusky and Kruskal [29] much attention is paid to the emergence of solitons and soliton trains from an
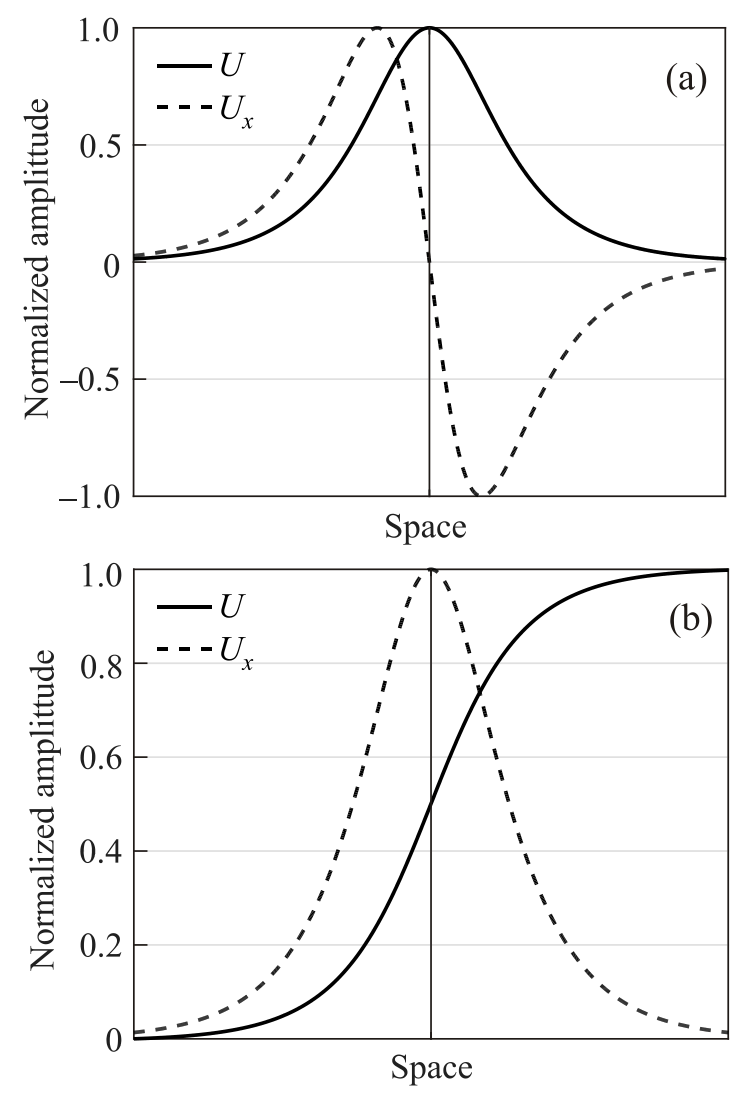

Fig. 1. (a) $\operatorname{sech}^{2}$-type displacement $U$ (solid line) and deformation $U_{X}$ (dashed line), (b) sech $^{2}$-type deformation $U_{X}$ (dashed line) and displacement $U$ (solid line). 


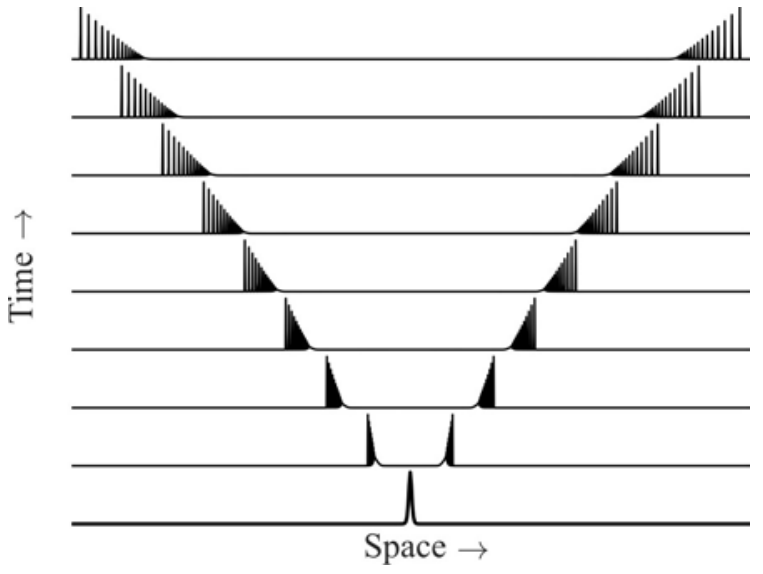

Fig. 2. Formation of trains of solitons from pulse-type initial condition for Eq. (9). Right- and left-going structures are plotted at every $\Delta T=2000$. For details see [24].

arbitrary input. Here we demonstrate main features of the emergence processes for both governing equations presented in Sec. 3. Note that there is a fundamental difference in solutions of these equations. Equation (9) describes the deformation while Eq. (10) describes the displacement. If we consider soliton-type solutions for both equations then there is a significant difference in displacements and deformations. A single pulse of a displacement means actually a sign-changing (bipolar) profile of a deformation (Fig. 1. (a)) and a single pulse of a deformation means a change of the displacement from one niveau to another (Fig. 1. (b)) [30].

The following results are obtained by numerical simulation using the pseudospectral method (see [31]) which gives high accuracy in computing. As far as the Boussinesq-type equations are bi-directional, from a localised initial input two soliton trains will emerge, one to the right, another to the left. Such a solution for the Eq. (9), i.e., the case of a solid with a microstructure is shown in Fig. 2 [24]. The initial input is taken

$$
V(X, 0)=A_{0} \operatorname{sech}^{2} B_{0}\left(X-X_{0}\right) .
$$

where $A_{0}$ is the amplitude, $B_{0}$ is related to the width of the pulse and $X_{0}$ defines the spacial shift of the input.

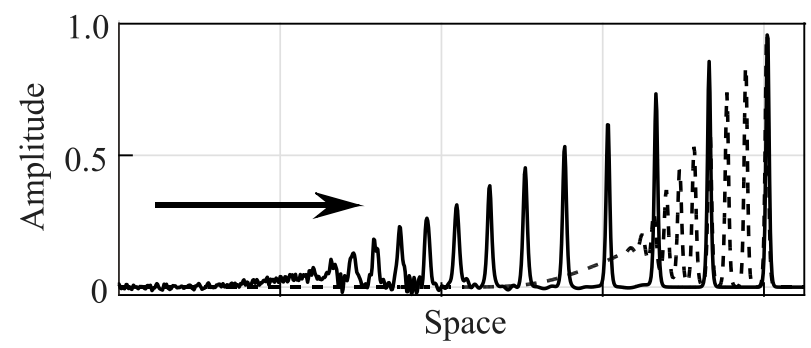

Fig. 3. Solutions of Eq. (9) in case of $A_{0}=1, B_{0}=0.01$ at dimensionless times $T=2230$ (dashed line) and $T=7000$ (solid line).

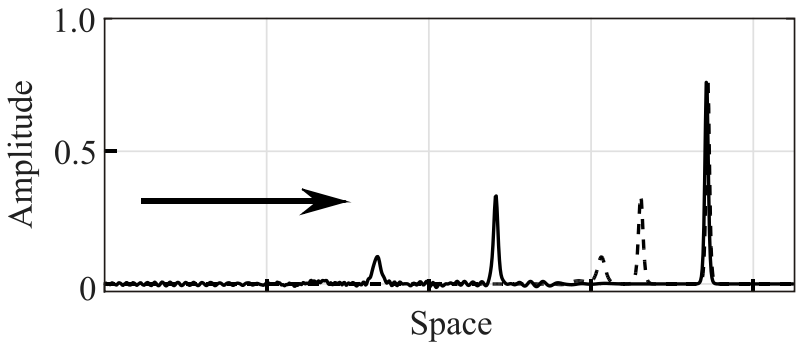

Fig. 4. Solutions of Eq. (9) in case of $A_{0}=1, B_{0}=0.05$ at dimensionless times $T=2230$ (dashed line) and $T=7210$ (solid line).

The coefficients here and further for solutions of Eq. (9) are taken $b=0.7188, \quad \mu=1.1395, \quad \delta=0.09, \quad \beta=45$, $v=9.3867, \lambda=1.1470$.

The number of solitons in a train depends on the energy of the initial pulse [24]. For example, two cases are shown in Figs. 3 and 4.

In the case of Eq. (10), i.e., the case of a biomembrane, the similar situation is observed. Here one should distinguish between the normal $\left(H_{1}<H_{2}\right)$ and anomalous $\left.\left(H_{1}>H_{2}\right)\right)$ dispersion. Given the values of $P<0$ and $Q>0$ [21] the following results are obtained [32]. Figure 5 demonstrates the emergence of soliton trains for anomalous dispersion and Fig. 6 - the emergence of soliton trains for normal dispersion.

The significant difference between the cases demonstrated above is the structure of a train. For microstructured solids the trains follow the conventional structure - the larger the amplitude of a soliton, the faster it propagates [29]. For biomembranes, however, given the values of nonlinear coefficients $(P<0$ and $Q>0)$ and anomalous dispersion, the outcome is different. In a train smaller solitons move ahead and larger solitons follow with a smaller speed. The comparison of two cases is demonstrated in Fig. 7.

\section{Summary}

The emergence of soliton trains is demonstrated for two cases of nonlinear and dispersive operators in the governing equations of the Boussinesq type for weakly dispersive media: microstructured solids and biomembranes. The

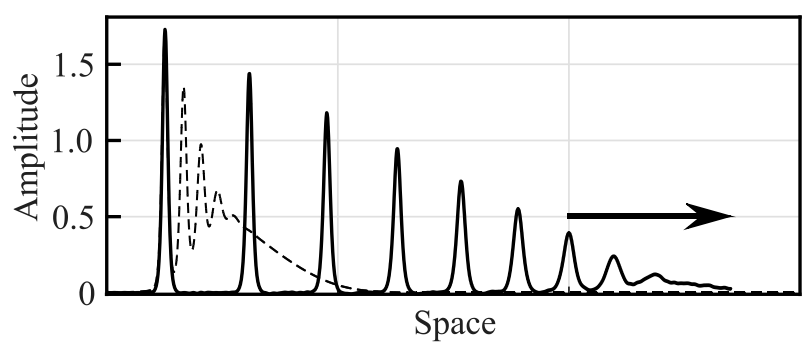

Fig. 5. Solutions of Eq. (10) in case of $P=-0.2186, Q=0.0043$, $H_{1}=0.072144, H_{2}=0.001$ at dimensionless times $T=15042$ (dashed line) and $T=98001$ (solid line). 


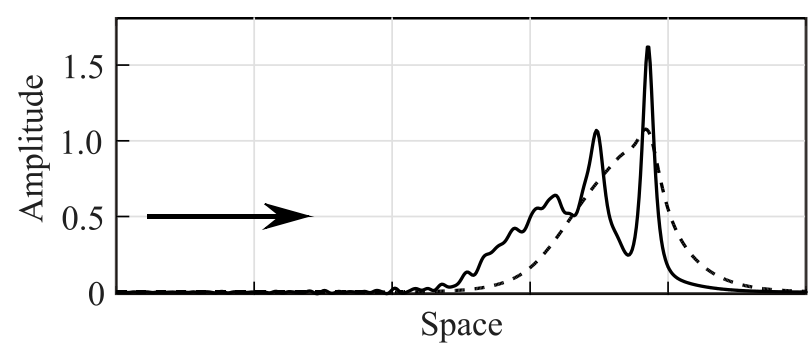

Fig. 6. Solutions of Eq. (10) in case of $P=-0.2186, Q=0.0043$, $H_{1}=0.072144, H_{2}=0.1$ at dimensionless times $T=21207$ (dashed line) and $T=98001$ (solid line).

dispersive effects in both cases are caused by the embedded microstructures while the competing nonlinearities are different. For microstructured solids, the nonlinearities are of the conventional deformation-type terms but for biomembranes due to the structure of bi-layers, the nonlinearities in terms of the governing wave equation are of the displacement-type. The corresponding governing equations allow variation over a wide range of values which results in the changes of nonlinear dynamics. The analysis demonstrates that both models permit the emergence of soliton trains. However, an important problem is to establish whether the single solitons in trains are 'pure' solitons or not. The studies reveal that the interactions of solitons obtained in both cases are not fully elastic. Referring to earlier studies out of scope of this paper [11,24], we note here that during the interactions the radiation occurs demonstrating the 'inelasticity' of interaction processes. So, strictly speaking, one should call the observed entities 'quasi-solitons' which is characteristic in many solitonic systems with inelastic interactions [3].
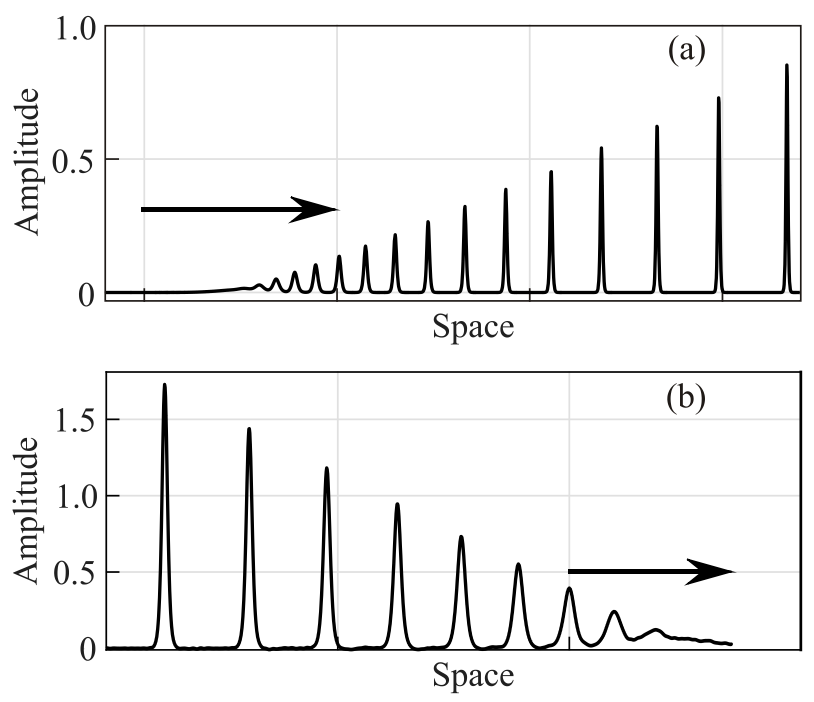

Fig. 7. (a) soliton train for Eq. (9); (b) soliton train for Eq. (10); arrows mark the direction of propagation.

\section{Acknowledgements}

This research was supported by the European Union through the European Regional Development Fund (Estonian Programme TK 124) and by the Estonian Research Council (projects IUT 33-24, PUT 434).

1. J. Boussinesq, Comptes Rendus l'Academie des Sci. 72, 755 (1871).

2. Lord Rayleigh, Philos. Mag. 1, 257 (1876).

3. C.I. Christov, G.A. Maugin, and A.V. Porubov, C. R. Mécanique 335, 521 (2007).

4. G.A. Maugin, Proc. Estonian Acad. Sci. Phys. Math. 44, 40 (1995).

5. G.A. Maugin, Nonlinear Waves in Elastic Crystals, Oxford University Press, Oxford (1999).

6. A.V. Porubov, Amplification of Nonlinear Strain Waves in Solids, World Scientific, Singapore (2003).

7. A. Berezovski, J. Engelbrecht, A. Salupere, K. Tamm, T. Peets, and M. Berezovski, Int. J. Solids Struct. 50, 1981 (2013).

8. A.M. Kosevich, The Crystal Lattice: Phonons, Solitons, Dislocations, Wiley-VCH, Berlin (1999).

9. A. Samsonov, Strain Solitons in Solids and How to Construct Them, Chapman and Hall/CRC, Boca Raton (2001).

10. J. Engelbrecht, F. Pastrone, M. Braun, and A. Berezovski, in: The Universality of Nonclassical Nonlinearity: Applications to Non-destructive Evaluations and Ultrasonics, P.P. Delsanto (ed.), p. 29 (2006).

11. J. Engelbrecht, K. Tamm, and T. Peets, Philos. Mag. 97, 967 (2017).

12. L.V. Bogdanov and V.E. Zakharov, Phys. D Nonlinear Phenom. 165, 137 (2002).

13. A.A. Himonas and D. Mantzavinos, J. Differ. Equ. 258, 3107 (2015).

14. M.M. Bogdan, A.M. Kosevich, and G.A. Maugin, Wave Motion 34, 1 (2001).

15. A. Kosevich and A. Kovalev, Solid State Commun. 12, 763 (1973).

16. O.V. Charkina and M.M. Bogdan, Symmetry, Integr. Geom. Methods Appl. 2, Paper 047 (2006).

17. G.A. Maugin and C.I. Christov, Proc. Estonian Acad. Sci. Physics. Math. 46, 78 (1997).

18. J. Engelbrecht, Nonlinear Wave Dynamics. Complexity and Simplicity, Kluwer, Dordrecht (1997).

19. A.C. Eringen, Nonlinear Theory of Continuous Media, McGraw-Hill Book Company, New York (1962).

20. G. Whitham, Linear and Nonlinear Waves, Wiley, New York (1974).

21. T. Heimburg and A.D. Jackson, Proc. Natl. Acad. Sci. USA 102, 9790 (2005).

22. R. Mindlin, Arch. Ration. Mech. Anal. 16, 51 (1964).

23. J. Engelbrecht, A. Berezovski, F. Pastrone, and M. Braun, Philos. Mag. 85, 4127 (2005). 
24. J. Engelbrecht, A. Salupere, and K. Tamm, Wave Motion 48, 717 (2011).

25. T. Peets, M. Randrüüt, and J. Engelbrecht, Wave Motion 45, 471 (2008).

26. J. Janno and J. Engelbrecht, Microstructured Solids: Inverse Problems, Heidelberg et al., Springer (2011).

27. J.K. Mueller and W.J. Tyler, Phys. Biol. 11, 051001 (2014).

28. I. Tasaki, Physiol. Chem. Phys. Med. NMR 20, 251 (1988).

29. S. Terakawa, J. Physiol. 369, 229 (1985).
30. J. Engelbrecht, K. Tamm, and T. Peets, Biomech. Model. Mechanobiol. 14, 159 (2015).

31. N. Zabusky and M. Kruskal, Phys. Rev. Lett. 15, 240 (1965).

32. T. Peets, K. Tamm, and J. Engelbrecht, Wave Motion 71, 113 (2017).

33. A. Salupere, in: Applied Wave Mathematics, E. Quak and T. Soomere (eds.), Springer Berlin Heidelberg, Berlin (2009), p. 301.

34. K. Tamm and T. Peets, Chaos, Solitons \& Fract. 73, 108 (2015). 